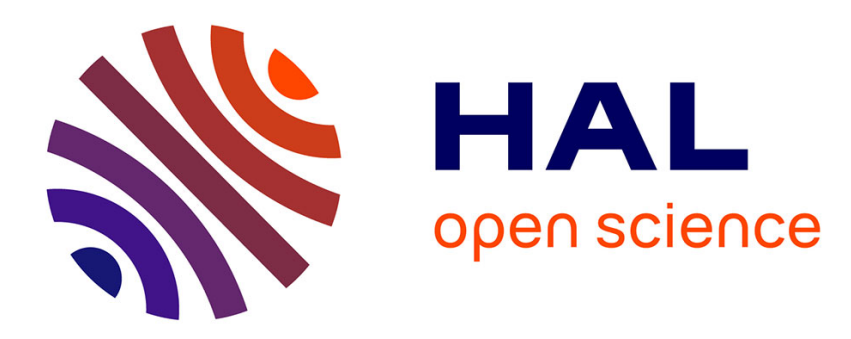

\title{
The Harmonic Tunneling Tag: a Dual-Band Approach to Backscattering Communications
}

\author{
Francesco Amato, Simon Hemour
}

\section{To cite this version:}

Francesco Amato, Simon Hemour. The Harmonic Tunneling Tag: a Dual-Band Approach to Backscattering Communications. 2019 IEEE International Conference on RFID Technology and Applications (RFID-TA), Sep 2019, Pisa, Italy. hal-02311456

\section{HAL Id: hal-02311456 https://hal.science/hal-02311456}

Submitted on 10 Oct 2019

HAL is a multi-disciplinary open access archive for the deposit and dissemination of scientific research documents, whether they are published or not. The documents may come from teaching and research institutions in France or abroad, or from public or private research centers.
L'archive ouverte pluridisciplinaire HAL, est destinée au dépôt et à la diffusion de documents scientifiques de niveau recherche, publiés ou non, émanant des établissements d'enseignement et de recherche français ou étrangers, des laboratoires publics ou privés. 


\title{
The Harmonic Tunneling Tag: a Dual-Band Approach to Backscattering Communications
}

\author{
Francesco Amato, Member, IEEE, and Simon Hemour, Senior Member, IEEE
}

\begin{abstract}
As an answer to the self-interference problem inherent to RFID systems, this work proposes a dual-band backscattering architecture. A dual-band RFID system architecture can bring several advantages to the RFID technology. In fact, a dual-band reader could operate without any self-interference cancellation circuit that currently limits its sensitivity; moreover, its dual-band architecture would be compatible with software defined radio-based cellphones. Dual-band readers will need to talk with low-powered dual-band tags that up-convert, modulate, and backscatter the impinging signal from the fundamental frequency to the $2^{\text {nd }}$ harmonic without weakening the communication link.

This article reports on the UHF dual-band Harmonic Tunneling Tag capable of backscattering and amplifying a $900 \mathrm{MHz}$ signal when receiving an input carrier at $450 \mathrm{MHz}$. The Harmonic Tunneling Tag provides positive conversion gains $(+7 \mathrm{~dB})$ that improve the communication link by a factor of 2 and operates under a very low bias voltage of $0.16 \mathrm{~V}$.

Index Terms-RFID, backscattering, tunnel diode, tunneling tag, reflection amplifier, harmonic tunneling tag, 5G, dual-band, UHF.
\end{abstract}

\section{INTRODUCTION}

$\mathbf{F}$ IFTH generation $(5 \mathrm{G})$ wireless communication systems will reshape the way cellphones are used and designed. Therefore, one of the opportunities that electrical engineers should not overlook is the possibility to embed RFID readers within the hardware of future $5 \mathrm{G}$ phones.

RFID, in fact, struggles in finding a widespread use in commercial applications other than supply-chains and in becoming as pervasive as Bluetooth Low Energy (BLE). One reason is that RIFD backscattering does not operate on two bands links as cellphones do requiring, therefore, a whole different hardware that does not integrate well within the cellphone architecture. Readers, moreover, by sending and receiving signals in the same frequency band, experience leakage of the transmitted carrier directly into the receiver causing extreme self-interference that limits their sensitivities and reduce the communication range.

Introducing in RFID systems the dual-band communication paradigm already used by cellphones would make the reader architecture less complex and open the possibility to integrate RFID readers into cellphones. Harmonic readers and tags can be the approach for developing dual-band RFIDs where the

Francesco Amato is with the Pervasive Electromagnetic Lab, Department of Civil and Computer Science Engineering, University of Roma Tor Vergata, 00133 Roma, Italy (e-mail: f.amato@ing.uniroma2.it); Simon Hemour is with the CNRS and IMS Research Center, Department of Science and Technology, University of Bordeaux, F-Talence, France (e-mail: simon.hemour@ubordeaux.fr).

978-1-7281-0589-5/19/\$31.00 (C)2019 IEEE. reader sends a signal at the fundamental frequency $f_{0}$ to interrogate a tag that reflects and modulates a higher harmonic (e.g.: $2 f_{0}$ ) detectable by the reader [1]. The transponder, therefore, requires a dual-band antenna and a non-linear device, such as a diode. Researchers have already explored the dual-band solutions for RFID transponders [2], [3], but the generation of harmonics comes with conversion losses that significantly affect the link performances. In fact, there are typically $30 \mathrm{~dB}$ of conversion losses at a typical input $\mathrm{RF}$ power of $1 \mu \mathrm{W}$.

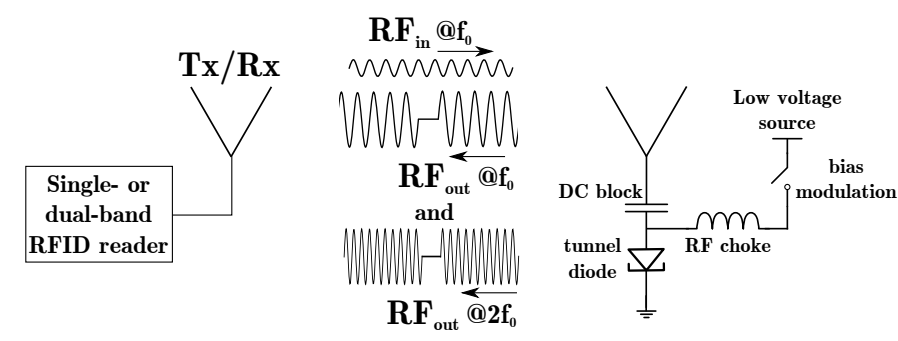

Fig. 1: A concept of the Harmonic Tunneling RFID tag communicating with either a dual-band or a single-band legacy RFID reader.

This work proposes to use a single tunnel diode to design low-powered dual-band RFID tags up-converting the input carrier frequency with positive conversion gain. The particular structure of a tunnel diode makes it suitable for different kind of applications; it can be used, for example, as impedance tuner [4] or as a reflection amplifier [5]. In RFIDs it can improve the read range [6] and allow the long-range tracking of tagged objects [7]. In this paper, we demonstrate the circuit design and preliminary measurement results of a Harmonic Tunneling Tag (Fig. 1) that uses non-linearities in tunnel diodes for generating amplified harmonics that improve the link budget and enables the deployment of dual-band RFID readers with no self-interference. The prototype uses tunnel diode AI301A [8] operating at $f_{0}=450 \mathrm{MHz}$ and backscattering an amplified signal $(+7 \mathrm{~dB})$ at $\operatorname{UHF}\left(2 f_{0}=900 \mathrm{MHz}\right)$ by using a very low biasing voltage. Although bulkier and less efficient than other tunnel diodes available in the market, the inexpensive and easy-to-buy tunnel diode AI301A from former soviet union was used for this work because of its high robustness that makes it suitable for early-stage tests and proof-of-concept development. 


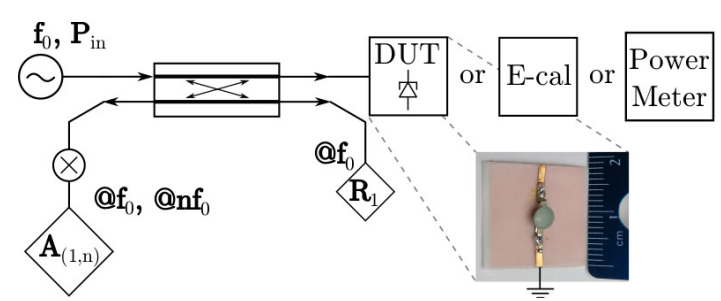

(a)

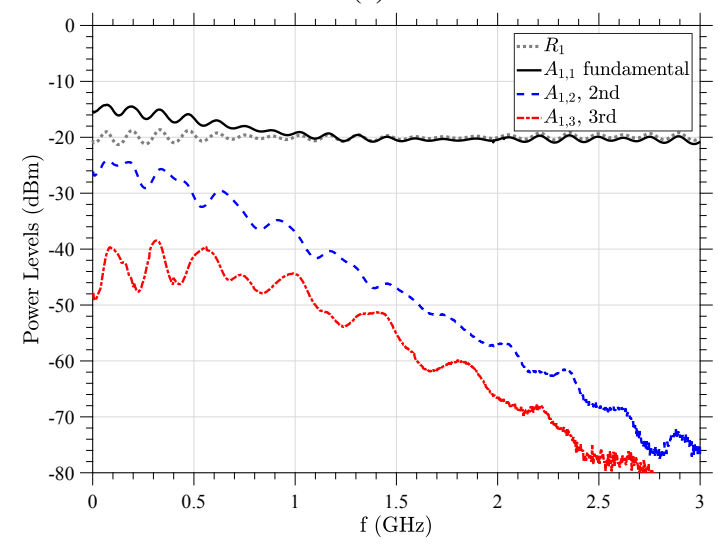

(b)

Fig. 2: a) Measurement setup with VNA E5080A. Inset: tunnel diode under test on substrate with $\epsilon_{r}=2.2$ and $30 \mu \mathrm{m}$ thickness. b) $R_{1}$ and $A_{1}$ at fundamental frequencies ( $f_{0} \in[1$ $\mathrm{MHz}, 3 \mathrm{GHz}]), A_{1,2}$ and $A_{1,3}$ of $2^{\text {nd }}\left(2 f_{0} \in\right.$ [2 MHz, $\left.\left.6 \mathrm{GHz}\right]\right)$ and $3^{\text {rd }}\left(3 f_{0} \in[3 \mathrm{MHz}-9 \mathrm{GHz}]\right)$ harmonics, respectively. Input power level $P_{i n}=-20 \mathrm{dBm}$ at power-calibrated port 1; $V_{\text {bias }}=0.16 \mathrm{~V}$.

\section{NON-LINEARITIES IN TUNNEL DiOdes}

To test the tunnel diode capabilities in reflecting and amplifying higher order harmonics VNA E5080A was used taking advantage of its frequency offset mode. Since the junction resistance and capacitance of the tunnel diode depend on the input signal power levels $P_{i n}$ and frequencies $f_{0}$, it is necessary to perform a full 1-port calibration as well as both source and receiver calibration. The block diagram in Fig. 2a summarizes the measurement setup: the power levels at source $R_{1}$ impinging on the tunnel diode at the fundamental frequencies $f_{0} \in[1 \mathrm{MHz}, 3 \mathrm{GHz}]$ were collected together with the power levels $A_{1, n}$ of the $n$-th reflected harmonics at frequencies $n f_{0}, n \in \mathbb{N}$ at the receiver. Fig. $2 \mathrm{~b}$ shows the impinging power level $R_{1}$ at the fundamental frequency range as well as the reflected power levels of both the fundamental frequencies $(n=1)$ and $2^{n d}$, and $3^{r d}$ harmonics for input power level $P_{i n}=-20 \mathrm{dBm}$. The reflected gains (or losses) of fundamental and higher order harmonics can be computed as $\frac{A_{1, n}}{R_{1}}$, with the $S_{11}$ parameter corresponding to the case of $n=1$.

Using a high precision DC power supply (E3648A) and multi-meter $34410 \mathrm{~A}^{1}$, the IV curve of tunnel diode in Fig. 3a

\footnotetext{
${ }^{1}$ Integration time defined in Number of Power Line Cycles, PLC $=10$
}

was measured. The curve allows to identify three main areas of interests: i) the tunneling effect region, in correspondence of no (or low) biasing voltages; ii) the non-linear regions at $0.16 \mathrm{~V}$; and iii) the negative resistance region at around $0.23 \mathrm{~V}$. The VNA was used to measure the generated reflected signals and harmonics of the diode shown in Fig. 2a; Fig. 3b, 3c, and $3 \mathrm{~d}$ show the reflected fundamental signals as well as the $2^{\text {nd }}$ and $3^{r d}$ harmonics. When no bias is applied (Fig. 3b), no amplification is observed and the harmonic levels are low. At the non-linear operating point of $0.16 \mathrm{~V}$ (Fig. 3d), as expected, higher gains and lower harmonics are measured. Despite the absence of any matching network (or matched antenna), these results already suggest a twofold use of the tunnel diode: by operating it at the non-linear point (e.g.: at $0.16 \mathrm{~V}$ ), it is possible to have a mixer/doubler behavior with conversion gain. By biasing it in the negative differential resistance region, instead, it can be used as an high-gain reflection amplifier for tunneling tags as already shown in [5].

\section{Harmonic Tunneling Tag Performance ESTIMATION}

By connecting a matching network (or a matched antenna) to the circuit while operating it in the non-linear region, it is possible to efficiently use the conversion gain to amplify the $2^{\text {nd }}$ harmonic and, hence, backscatter an amplified signal at frequency $2 f_{0}$ higher than the impinging one. This configuration will avoid the reader self-interference, will increase its sensitivity and will enable its integration in future dualband $5 \mathrm{G}$ cellphones. To verify this claim, the VNA was set to transform the fixture impendance to $23+\mathrm{j} 50 \Omega$, which is the conjugate impedance of the diode. The reflected signals at both fundamental and $2^{\text {nd }}$ harmonic frequencies were measured with an input power at the VNA port of $-30 \mathrm{dBm}$. As shown in Fig. 4, at $0.16 \mathrm{~V}$ bias, both the fundamental at $450 \mathrm{MHz}$ and the $2^{\text {nd }}$ harmonic at $900 \mathrm{MHz}$ are amplified with gains of $35 \mathrm{~dB}$ and $7 \mathrm{~dB}$, respectively. Of significant importance is the behaviour of the $2^{\text {nd }}$ harmonic: differently from other reported cases, where the frequency up-conversion comes at the expenses of severe conversion losses, the tunnel diode brings positive conversion gains. The amplified backscattered signals show how a Harmonic Tunneling Tag can either operate at the desired fundamental frequency $f_{0}$ or operate in dual-frequency mode and backscatter frequency up-converted signals without any conversion losses.

Using the free-space monostatic link budget equations [9], it is possible to compare the improved forward and backward distances with those of a traditional monostatic UHF RFID link for semi-passive tags mounting chip EM4325 [10]:

$$
P_{T}=P_{i n} G_{R} G_{t} \tau X\left(\frac{\lambda_{f}}{4 \pi r_{f}}\right)^{2}
$$

and

$$
P_{R}=P_{T} G_{R} G_{t} M X\left(\frac{\lambda_{b}}{4 \pi r_{b}}\right)^{2}
$$

assuming perfect matching $(\tau=1)$, no polarization mismatch $(X=1)$. With $P_{T}$ being the transponder circuit sensitivity, 


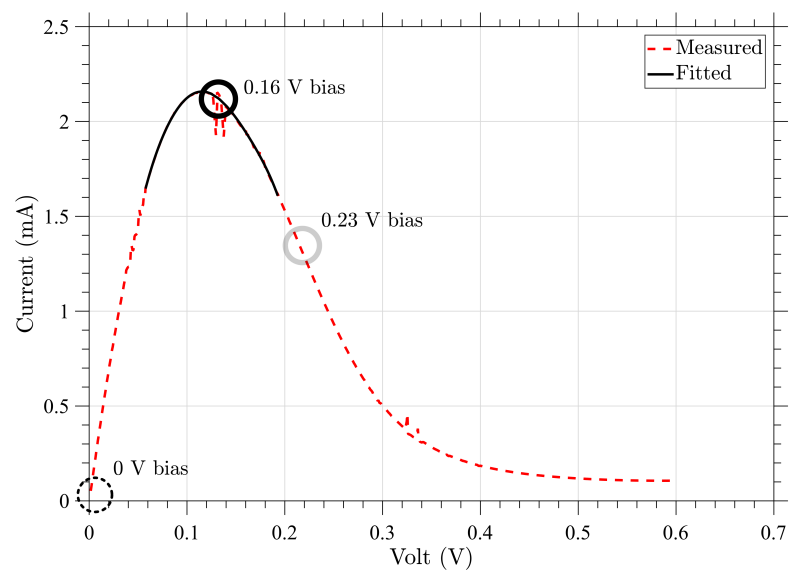

(a)

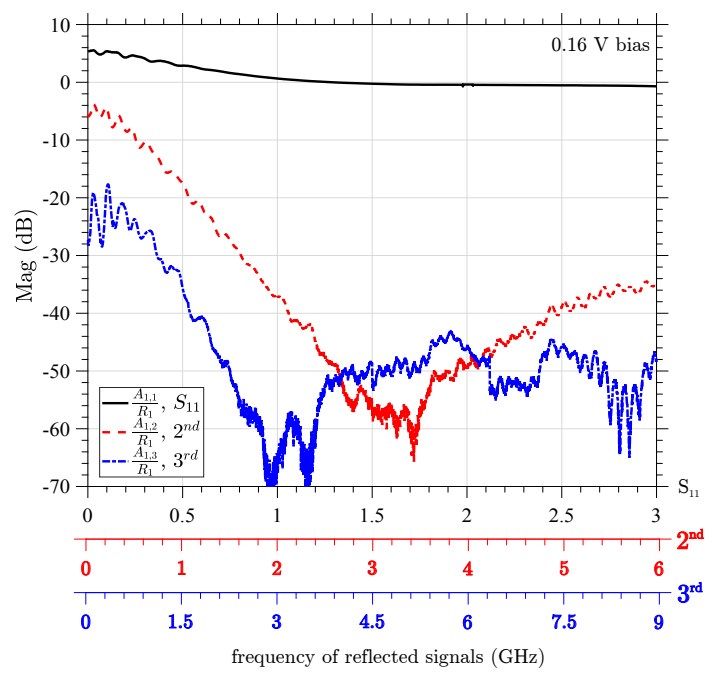

(c)

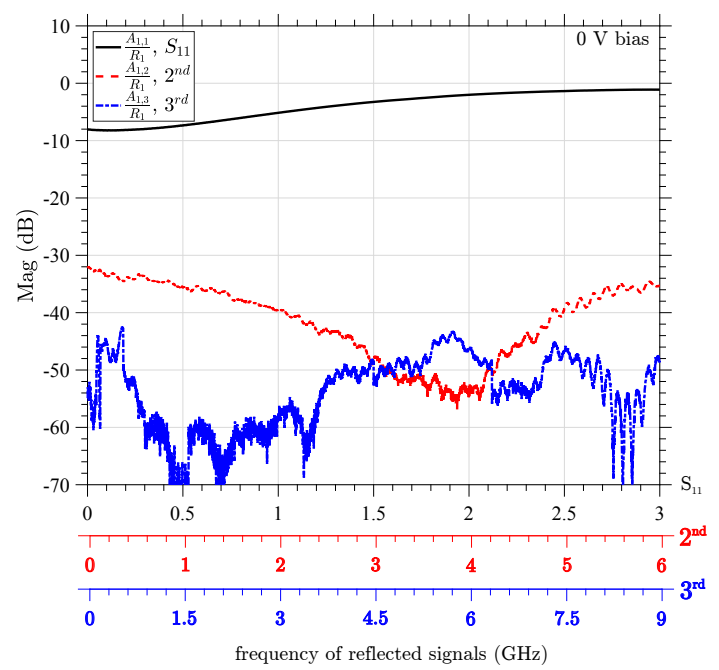

(b)

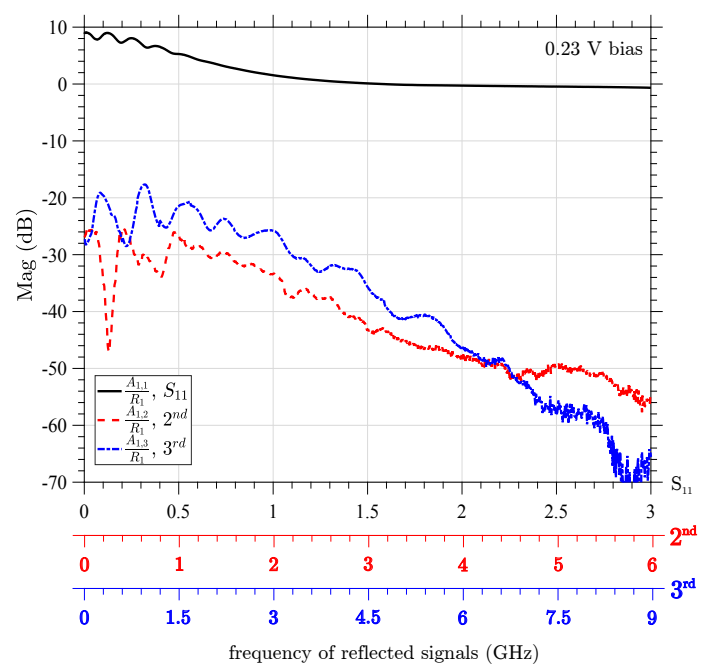

(d)

Fig. 3: a) Measured and Fitted Tunnel Diode IV curve. $A_{1, n} / R_{1}$ data for different biasing conditions: b) 0 V; c) 0.16 ; and d) $0.23 \mathrm{~V}$.

$P_{i n}$ being the power transmitted by the reader, $M$ being the modulation factor, $G$ denoting the antenna gains, $\lambda_{f / b}=\frac{c}{f_{f / b}}$ being the operation wavelengths and $r_{f / b}$ the maximum achievable distances in either the forward or the backward links. The maximum achievable distances $r_{\max }$ are limited by the minimum between the forward and the backward links: $r_{\max }=\min \left(r_{f}, r_{b}\right)$. A comparison can be made between a semi-passive UHF tag and the Harmonic Tunneling Tag (HTT) operating in dual-band mode. The parameters used for the comparison are listed in Tab. I while Tab. II reports the forward and backward distances for the two tag architectures. The Harmonic Tunneling Tag in dual-band mode, improves the read distance of backward link-limited semi-passive tags from 10 to 18 meters. It is important to point out that these distances were computed assuming a low dual-band reader sensitivity that should actually improve for a dual-band selfinterference-free RFID system. Moreover, the biasing voltage
TABLE I: Link budget parameter values

\begin{tabular}{|c|c|c|c|c|}
\hline \hline & & UHF Tag & HTTs \\
\hline \hline$P_{i n}$ & $30 \mathrm{dBm}$ & & Ref. [10] & Dual-band \\
\cline { 3 - 5 }$G_{R}$ & $6 \mathrm{dBi}$ & & 0 & 7 \\
$G_{t}$ & $0 \mathrm{dBi}$ & $P_{T}(\mathrm{dBm})$ & $-28^{*}$ & -30 \\
$P_{R}$ & $-74 \mathrm{dBm}[11]$ & $f(\mathrm{MHz})$ & 900 & $450 / 900$ \\
\hline \hline
\end{tabular}

required for the Harmonic Tunneling Tag is about one order of magnitude lower than the bias required by the current semipassive ICs.

\section{CONCLUSIONS}

This article demonstrated how tunnel diodes can significantly contribute in revolutionising the UHF RFID technology. In fact, a biasing voltage as low as $0.16 \mathrm{~V}$ can trigger tunnel 


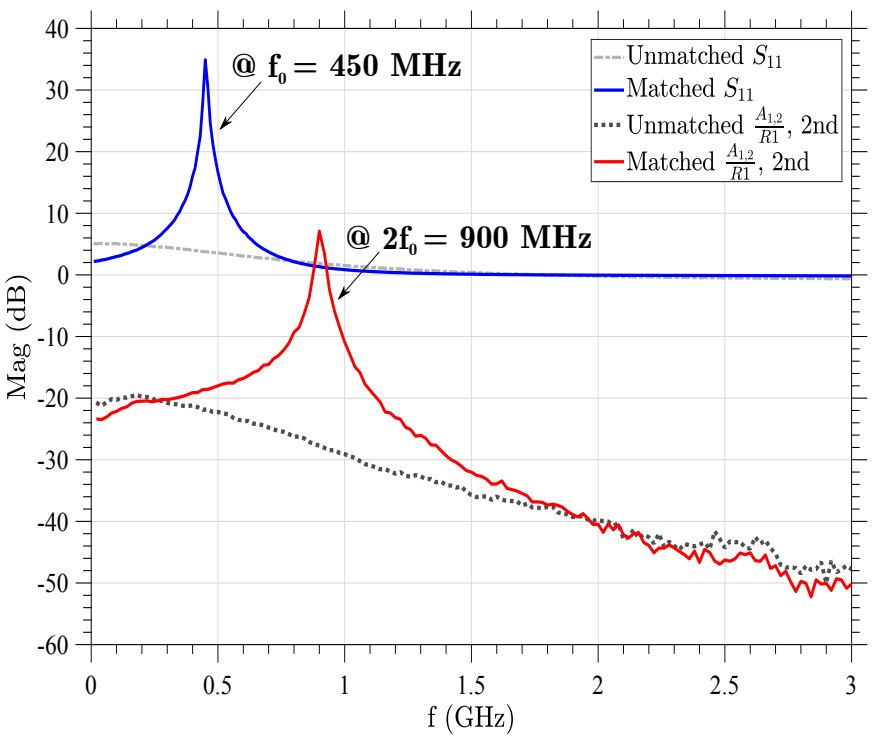

Fig. 4: Return $\left(S_{11}\right)$ and conversion gains $\left(\frac{A_{1,2}}{R_{1}}\right)$. Dotted gray lines: gains measured on a $50 \Omega$ port VNA (with frequency offset). Solid colored lines: computation by the VNA of fundamental return gains $(+35 \mathrm{~dB}$ at $450 \mathrm{MHz})$ and $2^{\text {nd }}$ harmonic conversion gain $(+7 \mathrm{~dB}$ at $900 \mathrm{MHz})$ if the power impedance is set to $23+\mathrm{j} 50 \Omega$. The VNA port power $P_{i n}$ is set to $-30 \mathrm{dBm}$, a $3 \mathrm{~dB}$ attenuation is used to reduce the loading effect of the negative resistance on the VNA generator. At the peak gain, the RF power efficiently reaching the tunnel junction is below $-60 \mathrm{dBm}$.

TABLE II: Achievable distances

\begin{tabular}{|c|c|c|}
\hline \hline & $\begin{array}{c}\text { UHF tag } \\
\text { Ref. [10] }\end{array}$ & $\begin{array}{c}\text { HTT } \\
\text { Dual Band }\end{array}$ \\
\hline$r_{f}(\mathrm{~m})$ & 42.1 & 106 \\
\hline$r_{b}(\mathrm{~m})$ & $\mathbf{1 0 . 6}$ & $\mathbf{1 8 . 8}$ \\
\hline \hline
\end{tabular}

diode non-linearities to backscatter amplified $2^{\text {nd }}$ harmonic frequencies at the UHF band. A Harmonic Tunneling Tag improves the link budget by up-converting and amplifying the reflected signal; moreover, it allows RFID readers to operate in dual-band mode avoiding the self-interference that currently degrades their sensitivity.

Tunnel diodes are not the only devices that can provide negative small signal resistance (a pair of transistors could fairly exhibit this behavior for the same package footprint). However, the extremely low bias voltage demonstrated in this work would be impossible to reach. To properly operate, in fact, the tunnel diode requires $0.16 \mathrm{~V}$ and draws a current of $2 \mathrm{~mA}$. Although the required current is already low, it is important to highlight that more efficient tunnel diodes operating at much lower voltages and currents are available in the market. This makes the Harmonic Tunneling Tags compatible with many ambient energy harvesting techniques [12], [13], [14] that could contribute in developing a completely passive long-range RFID tag.

The Harmonic Tunneling Tag brings two important advan- tages to the UHF RFID technology, both in the short and in the long term. In fact, the added gains of the $2^{\text {nd }}$ harmonic (instead of conversion losses) would make it possible to easily integrate dual band RFID readers within current and nextgen cellphones that typically operate in dual band to mitigate interference.

Moreover, the possibility of amplifying and backscatter the impinging main carrier, further demonstrates how Tunneling Tags can be used to improve both the current UHF RFID technology and future RFIDs operating either at UHF, or at microwave (e.g.: $5.8 \mathrm{GHz}$ ) or at mm-Wave (e.g.: $60 \mathrm{GHz}$ ) frequencies.

\section{ACKNOWLEDGEMENTS}

The authors would like to thank Prof. P. Fay, University of Notre Dame, for the fruitful discussions.

\section{REFERENCES}

[1] V. Palazzi, F. Alimenti, C. Kalialakis, P. Mezzanotte, A. Georgiadis, and L. Roselli, "Highly integrable paper-based harmonic transponder for low-power and long-range iot applications," IEEE Antennas and Wireless Propagation Letters, vol. 16, pp. 3196-3199, 2017.

[2] X. Gu, S. Nikhil N, L. Guo, S. Hemour, and K. Wu, "Diplexer-based fully passive harmonic transponder for sub-6-GHz 5G-compatible IoT applications," IEEE Transactions on Microwave Theory and Techniques, vol. 67, no. 5, pp. 1675-1687, May 2019.

[3] P. V. Nikitin and K. V. S. Rao, "Harmonic scattering from passive uhf rfid tags," in 2009 IEEE Antennas and Propagation Society International Symposium, June 2009, pp. 1-4.

[4] Y. Zhao, S. Hemour, T. Liu, and K. Wu, "Negative resistance-based electronic impedance tuner," IEEE Microwave and Wireless Components Letters, vol. 28, no. 2, pp. 144-146, Feb 2018.

[5] F. Amato, C. W. Peterson, B. P. Degnan, and G. D. Durgin, "Tunneling RFID tags for long-range and low-power microwave applications," IEEE Journal of Radio Frequency Identification, vol. 2, no. 2, pp. 93-103, June 2018.

[6] F. Amato, H. M. Torun, and G. D. Durgin, "RFID backscattering in long-range scenarios," IEEE Transactions on Wireless Communications, vol. 17, no. 4, pp. 2718-2725, April 2018.

[7] C. Qi, F. Amato, M. Alhassoun, and G. D. Durgin, "Breaking the range limit of RFID localization: Phase-based positioning with tunneling tags," in 2019 IEEE International Conference on RFID (RFID), April 2019, pp. $1-8$.

[8] "Tunnel diode AI301A," 2019 (Accessed May 25, 2019). [Online]. Available: https://eandc.ru/pdf/diod/ai301.pdf

[9] J. Griffin and G. Durgin, "Complete link budgets for backscatter-radio and RFID systems," Antennas and Propag. Magazine, IEEE, vol. 51, no. 2, pp. 11-25, Apr. 2009.

[10] EMmicroelectronic, "EM4325," 2019 (Accessed May 25, 2019). [Online]. Available: https://www.emmicroelectronic.com/sites/default/ files/products/datasheets/4325-ds.pdf

[11] Impinj, Apr. $2019 \quad$ (Accessed May 25, 2019). [Online]. Available: https://support.impinj.com/hc/en-us/articles/ 204969718-Indy-RS2000-Datasheet

[12] Z. H. Zhang, Y. S. Gui, L. Fu, X. L. Fan, J. W. Cao, D. S. Xue, P. P. Freitas, D. Houssameddine, S. Hemour, K. Wu, and C.-M. Hu, "Seebeck rectification enabled by intrinsic thermoelectrical coupling in magnetic tunneling junctions," Phys. Rev. Lett., vol. 109, p. 037206, Jul 2012. [Online]. Available: https://link.aps.org/doi/10.1103/PhysRevLett. 109.037206

[13] C. H. P. Lorenz, S. Hemour, W. Li, Y. Xie, J. Gauthier, P. Fay, and $\mathrm{K}$. Wu, "Breaking the efficiency barrier for ambient microwave power harvesting with heterojunction backward tunnel diodes," IEEE Transactions on Microwave Theory and Techniques, vol. 63, no. 12, pp. 4544-4555, Dec 2015.

[14] G. D. Durgin, "RF thermoelectric generation for passive RFID," in 2016 IEEE International Conference on RFID (RFID), May 2016, pp. 1-8. 